\title{
Time evolution of chemistry with fixed physical parameters in TMC-1
}

\section{Peter Berczik ${ }^{1,3}$, Peter Bertsyk ${ }^{2,4}$, Orsolya Fehér ${ }^{2}$, Jorma Harju ${ }^{5}$, Toshikazu Onishi ${ }^{6}$ and L. Viktor Tóth ${ }^{2}$}

\author{
${ }^{1}$ National Astronomical Observatories of China, Chinese Academy of Sciences, Beijing, China \\ ${ }^{2}$ Department of Astronomy, Lorand Eotvos University, Budapest, Hungary. \\ ${ }^{3}$ National Academy of Sciences of Ukraine, Main Astronomical Observatory, Kyiv, Ukraine. \\ ${ }^{4}$ Department of Physical Chemistry, Lorand Eotvos University, Budapest, Hungary \\ email: bertsykpeter@caesar.elte.hu. \\ ${ }^{5}$ Department of Physics, University of Helsinki. \\ ${ }^{6}$ Dept. of Physical Science, Osaka Prefecture University, Osaka, Japan.
}

\begin{abstract}
We present a set of time dependent chemical evolution models (based on the UMIST $\dagger$ astrochemistry 2012 code, Woodall et al. (2007); McElroy et al. (2013) for a range of initial physical cloud parameters: $10 \mathrm{~K}<\mathrm{T}<20 \mathrm{~K} ; 10^{3} \mathrm{~cm}^{-3}<\mathrm{n}\left(\mathrm{H}_{2}\right)<5 \cdot 10^{4} \mathrm{~cm}^{-3} ; 1<\mathrm{AV}<10$ and with estimated values of scaled interstellar ultraviolet radiation field. Our computation model included the full UMIST gas-phase reaction network for 467 species Garrod et al. (2008), Graedel et al. (1982). We compare our chemical model results with the relative abundances of: $\mathrm{CO}, \mathrm{CH}$, $\mathrm{OH}, \mathrm{HCO}^{+}, \mathrm{HCN}, \mathrm{HNC}, \mathrm{NH}_{3}, \mathrm{~N}_{2} \mathrm{H}^{+}$and $\mathrm{H}_{2} \mathrm{CO}$ molecules. We find significant time dependent variations of the chemical ratios of: $\mathrm{X}\left(\mathrm{NH}_{3} / \mathrm{H}_{2}\right) ; \mathrm{X}\left(\mathrm{HCO}^{+} / \mathrm{H}_{2}\right)$ and $\mathrm{X}\left(\mathrm{HCO}^{+} / \mathrm{NH}_{3}\right)$. We derive an ammonia age spread for the parts of TMC-1 (Taurus Molecular Cloud-1) that looks more complex than previous estimated showed. Age estimates based on $\mathrm{X}\left(\mathrm{NH}_{3} / \mathrm{H}_{2}\right) ; \mathrm{X}\left(\mathrm{HCO}^{+} / \mathrm{H}_{2}\right)$ and $\mathrm{X}\left(\mathrm{HCO}^{+} / \mathrm{NH}_{3}\right)$ were compared in 3 selected positions, and were found to be very similar (with $\pm 9 \%$ differences).
\end{abstract}

Keywords. Keyword1, keyword2, keyword3, etc.

\section{Modeling TMC-1}

Time dependent variations of the abundance ratios of: $\mathrm{X}\left(\mathrm{NH}_{3} / \mathrm{H}_{2}\right) ; \mathrm{X}\left(\mathrm{HCO}^{+} / \mathrm{H}_{2}\right)$ and $\mathrm{X}\left(\mathrm{HCO}^{+} / \mathrm{NH}_{3}\right)$ were estimated with our chemical modeling. Using the same time dependent chemical modeling, we can also roughly estimate in each observed point the best corresponding "chemical age". The input physical conditions, for the observed points, were taken from Fehér O. et al. (2015) at 16 positions along the TMC-1 dark cloud. As Figure 1 shows the ammonia and formyl ion abundances show a considerable increase in few hundred kyrs in the given range of density, extinction and temperature. The $\mathrm{NH}_{3}$ and $\mathrm{HCO}^{+}$relative densities based on the observations of Fehér O. et al. (2015) and Onishi et al. (2002) help to find the "chemical age" of the given position in the evolution curves.

In Table 1 we lists the 5 positions along the TMC- 1 ridge with the derived ages. The observed $\mathrm{NH}_{3}(1,1)$ line intensity is presented in Figure 2 (gray scale) with positions where "chemical ages" was estimated (shown with color circles, see also Figure 1, and Table 1). We note that there is a "young" southern part and a more "older" northern part with a mixed chemical age. Based on our modeling we can conclude that for the selected observational points we can get the quite consistent ages. We can see this as a independent proof of our chemical age determination.

Acknowledgments: PB acknowledge the special support by the NASU under the Main Astronomical Observatory GRID/GPU computing cluster GOLOwOOD project. PB acknowledge the financial support by National Astronomical Observatories, Chinese Academy of Sciences, funded by the Ministry of Finance of People's Republic of China under the grant ZDYZ2008-2.

$\dagger$ UMIST Database for Astrochemistry can be downloaded from the following link: http://www .udfa.net 

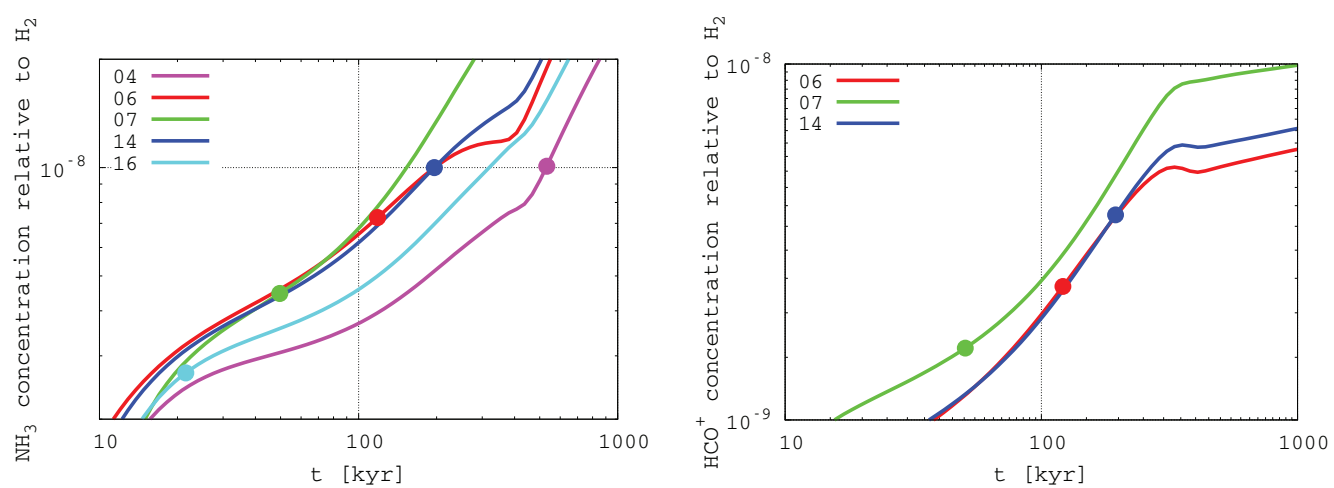

Figure 1. Time dependent variations of the abundance ratios as a function of time.

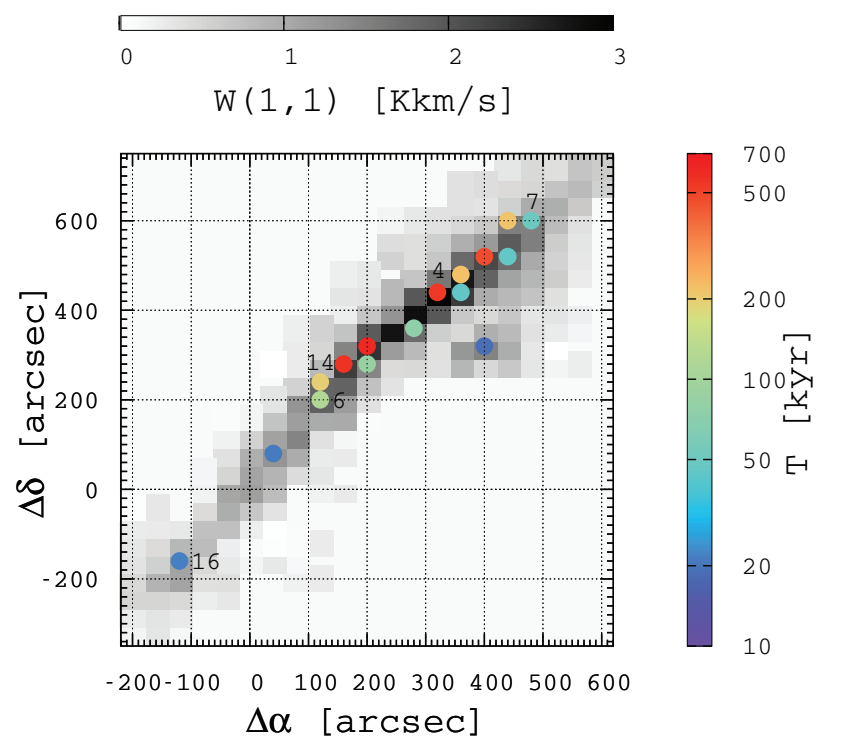

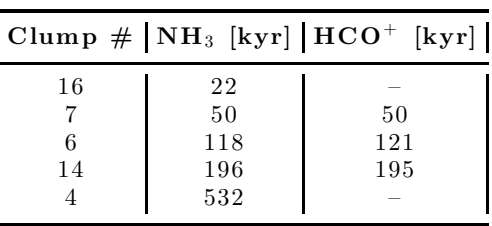

Figure 3. 5 positions with estimated ages.

Figure 2. Observed $\mathrm{NH}_{3}(1,1)$ line intensity.

This research was partly supported by the OTKA K101393 and NN-111016 grants. P. Bertsyk would like to acknowledge his scientific adviser D.Sc. Baranyai András for constant help and support.

\section{References}

Fehér O., Ward-Thompson D., Tóth L.V., Kirk J., Pelkonen V.M., Zahorecz S. \& Pintér S. 2015, $A \mathscr{E} A$, submitted

Garrod R.T., Weaver S.L.W., \& Herbst E. 2008, ApJ, 682, 283

Graedel T.E., Langer W.D., \& Frerking M.A. 1982, ApJS, 48, 321

McElroy D., Walsh C., Markwick A.J., Cordiner M.A., Smith K., Millar T.J. 2013, A\& A, 550, A36

Onishi T., Mizuno A., Kawamura A., Tachihara K. \& Fukui Y. 2002, ApJ, 575, 950

Woodall J., Agndez M., Markwick-Kemper A.J., Millar T.J. 2007, A\&\&A, 466, 1197 